\title{
Investigation Studies on the Application of Reverberation Time
}

\author{
Artur NOWOŚWIAT, Marcelina OLECHOWSKA
}

Faculty of Civil Engineering, Silesian University of Technology

Akademicka 5, 44-100 Gliwice, Poland; e-mail: \{artur.nowoswiat, marcelina.olechowska\}@polsl.pl

(received February 12, 2015; accepted November 25, 2015)

\begin{abstract}
The paper presents the research studies carried out on the reverberation time of rooms, in terms of theoretical aspects and applicability potentials. Over the last century a very large number of scientists have been attempting to work out models describing the reverberation time in enclosed rooms. They have also been trying to apply these models for the description of various acoustic parameters of the interior, i.e. the intelligibility of speech, clarity, articulation, etc. In fact, all these models are based on the Sabine's statistical method. The paper presents the work of the scientists working on this problem, together with prospective applicability potentials. Such a review may be helpful for researchers, designers or architects involved in the discussed subject.
\end{abstract}

Keywords: reverberation time; research studies; Sabine.

\section{Introduction}

In the early twentieth century Wallace Clement Sabine developed a statistical model based on the statistical theory of sound field. It assumed that the density of sound energy at any point of the interior was equal, and the probability of incidence of the sound wave was the same in all directions. The model uses the concepts of mean free path length of sound waves and average waveform time. According to the statistical theory, the decay process of sound energy in the room, after turning off the stationary sound source, is of exponential nature, and the parameter of such a decay is expressed by a constant quantity, i.e. reverberation $(T-$ reverberation time). That method was applied to formulate the generally known Sabine and Eyring formulas, which are now used to determine the reverberation time of the interior with the known volume and sound absorption of the room. In the statistical theory of sound field in a room, Sabine described the phenomenon of reverberation, and also, basing on the results of his research, he provided the empirical (primary) formula to calculate reverberation time, which has the following form (SABINE, 1922):

$$
T_{S A B}=\frac{0.161 V}{S \bar{\alpha}_{S A B}}[\mathrm{~s}], \quad \bar{\alpha}_{S A B}=\frac{1}{S} \sum_{i=1}^{n} \alpha_{i} S_{i} .
$$

Taking into account sound isolation effected by transfer in the air, we obtain the following equation:

$$
T_{S A B}=\frac{0.161 V}{S \bar{\alpha}_{S A B}+4 m V}[\mathrm{~s}]
$$

where $T_{S A B}$ - reverberation time acc. Sabine $[\mathrm{s}], V-$ volume of the room, $S$ - total internal surface area of the room $\left[\mathrm{m}^{2}\right], \bar{\alpha}_{S A B}$ - average absorption coefficient, $m$ - air sound absorption coefficient $\left[\mathrm{Np} \cdot \mathrm{m}^{-1}\right]$.

Many empirical equations have been formulated for rectangular rooms. The first one was offered in the work of (Fitzroy, 1959), then (Pujolle, 1975; HIRAtA, 1979), but also (Neubauer, Kostek, 2001), or (ARAu-PuCHADES, 2005) and others. The said scientists presented different approach to their empirical equations. Fritzoy assumed the arithmetic average of reverberation time in three orthogonal directions. On the other hand the Arau-Puchades formula favours the geometric average. Both mentioned equations are simple in their assumptions but they do not prove efficient in more complicated rooms. Hirata based his reverberation theory on the distribution of acoustic field in one-, two- and three-dimensional areas dependant on frequency. In other words, the increase of scattering field results in the decrease of reverberation time. Yet the said effect strongly depends on the shape and sound absorption of the room (LAM, 1996; WANG, RATHSAM, 2008). Measurement methods which allow for a scattered field are presented in the Standard 
ISO 17497-1. Unfortunately, there are not many theories in room acoustics which allow for uneven scattering of sound in a room. Such an approach was proposed by SAKUMA (2012). In his work he proposed, first of all, that an approximate theory of reverberation in rectangular rooms should be formulated as a specular reflection field based on the image source method by modifying Hirata's theory. Secondly, considering surface scattering on walls with scattering coefficients, an integrated reverberation theory for nondiffuse field should be developed, where the total field is divided into specular and diffuse reflection fields. Finally, he obtained a theoretical case study demonstrating how surface scattering affects the energy decay of nondiffuse fields in rectangular rooms, when changing the aspect ratio and the absorption distribution.

Reverberation time is also widely applied for the estimation of other acoustic parameters.

The results of many research studies indicate (Plomb et al., 1980; Houthast, Steeneken, 1984; 1985; Ozimek, Rutkowski, 1985) that apart from the level of speech signal or the interference level of amplitude-time structure of sound reflection, the intelligibility of speech in a room is highly dependent on reverberation time. On the other hand, GALBRUN and KITAPCI (2014) investigated the accuracy of speech transmission index as dependent on reverberation time and on the signal-noise ratio.

Many research studies demonstrate that by the application of reverberation time we can quickly estimate various parameters or indexes describing room acoustics. Such an approach was pursued by Lam. In his work (LAM, 1999), using only reverberation time, he worked out a method for the estimation of Deutlichkeit, clarity and center time. In the same way Nowoświat and Olechowska (2016), using the statistical analysis, worked out the estimation method of STI, applying for that purpose reverberation time and BISTAFA and BRADLEY (2000) plotted STI values versus reverberation time for unamplified speech in classrooms.

At present, when designing lecture rooms, concert halls, auditoriums, etc., reverberation time is definitely taken into account. ArETz and OrLOWski (2009) demonstrated in their measurements that for a definite volume of a room, sound strength can be decreased by means of an appropriate alteration of reverberation time. But on the other hand, to ensure proper reception of chamber music, reverberation time cannot be decreased freely. Therefore, they suggested that reverberation time and sound strength should be balanced. Basing on the least squares method, they worked out a regression model.

Reverberation time is also important in shaping the acoustics of sacral interiors. For example, BERARDI (2012) and earlier ENGEL and KosatA (2007) applied reverberation time among other things for a single in- dicator rating of such interiors. Wide application of reverberation time in shaping the acoustics of interiors instigated the authors to describe in one paper more or less known models and theories involving it.

\section{Theoretical models of reverberation time}

The reverberation time of a room can be calculated using the empirical formulas described in the works of (ARAu-PuChADES, 1988; Eyring, 1930; Fitzroy, 1959; Millington, 1932; Neubauer, Kostek, 2001; Pujolle, 1975; SABIne, 1922; Sette, 1933). Equation (1) for the prediction of reverberation time shows that the reverberation time of a room can be determined basing on the interior volume $V$ and on its sound absorption $A$. The Sabine's formula is very simple, but it is also burdened with some limitations. The formula gives wrong results for completely soundproof rooms $\left(\bar{\alpha}_{S A B}=1\right)$. Instead of the expected $T=0$, the value of reverberation time different from zero is obtained for the room interior. Therefore, the formula derived by Sabine is designed for poorly soundproof rooms $\left(\bar{\alpha}_{S A B}<0.2\right)$ with evenly distributed sound absorption. This formula has historical significance, and with the development of science, it has given rise to several transformations which are free from such limitations. Additionally, a modification has been introduced into the formulas for rooms of the volume larger than $200 \mathrm{~m}^{3}$ which allows for the effect of air absorption (PN EN 12354-6, 2005). A modified determination method of reverberation time was proposed by Norris and Eyring (EYrInG, 1930; NorRIs, AndrEe, 1930). When they were expanding the calculation concept for sound absorption coefficient given by SABINE (1922), they introduced a logarithmic dependence for the average coefficient $\alpha$ into the denominator. They presented the converted Sabine's formula which is devoid of the limitation mentioned above and takes the following form:

$$
T_{E Y R}=\frac{0.161 V}{S \bar{\alpha}_{E Y R}}[\mathrm{~s}], \quad \bar{\alpha}_{E Y R}=-\ln \left(1-\bar{\alpha}_{S A B}\right),
$$

Knudsen modified the Eyring's formula by introducing the isolation of sound in the air into Eq. (3) (KNUDSEN, 1929):

$$
T_{E Y R}=\frac{0.161 V}{S \bar{\alpha}_{E Y R}+4 m V}[\mathrm{~s}] .
$$

The formula introduced by Eyring, in contrast to the Sabine's formula, can be used for all values of the mean sound absorption coefficient. If the mean sound absorption coefficient is small $\left(\bar{\alpha}_{S A B}<0.2\right)$, the development of the denominator in the expression (3) into Taylor's sequence is equal to $-\ln \left(1-\bar{\alpha}_{S A B}\right)=\bar{\alpha}_{S A B}$, and then the formula takes the form of Sabine's formula. In such a case, the relative difference $\Delta T$ of the interior reverberation time calculated with the use of 
the above formulas does not exceed $9 \%$. The difference is brought about by omitting further terms of the sequence in the Sabine's formula. The value of $\Delta T$ increases with the rise of $\bar{\alpha}_{S A B}$, and thus the scope of applicability of the Sabine's formula is reduced to the values of the coefficient $\left(\bar{\alpha}_{S A B}<0.2\right)$ :

$$
\begin{aligned}
\Delta T & \cong \frac{\frac{0.16 V}{S \bar{\alpha}_{S A B}}-\frac{0.16 V}{-S\left(1-\bar{\alpha}_{S A B}\right)}}{\frac{0.16 V}{S \bar{\alpha}_{S A B}}} \cdot 100 \% \\
& =\frac{\ln \left(1-\bar{\alpha}_{S A B}\right)+\bar{\alpha}_{S A B}}{\ln \left(1-\bar{\alpha}_{S A B}\right)} \cdot 100 \% \\
& =\frac{-0.22+0.2}{0.22} \cdot 100 \%=9 \% .
\end{aligned}
$$

Another formula was presented by MiLlington (1932) and SeTte (1933). Their formula differs from the previously described formulas in the determination method of the average sound absorption coefficient. In the Sabine's formula, the coefficient $\bar{\alpha}_{S A B}$ is determined as the arithmetic mean, whereas Millington suggested the calculation of the coefficient $\bar{\alpha}_{M I L}$ as the geometric mean. The Millington's formula cannot be used in perfectly absorbing rooms, since in such a case indeterminacy is obtained in the denominator:

$$
\begin{aligned}
T_{M I L} & =\frac{0.161 V}{S \bar{\alpha}_{M I L}}[\mathrm{~s}], \\
\bar{\alpha}_{M I L} & =-\frac{1}{S} \sum_{i=1}^{n} S_{i} \ln \left(1-\alpha_{i}\right) .
\end{aligned}
$$

KuttrufF (2009), on the other hand, suggested in his work a statistical distribution of sound, taking into account the Gaussian random variable and Rayleigh probability. Basing on that concept, he created a definition of the function of mean free path $\gamma^{2}=\left(\overline{l^{2}}-\bar{l}^{2}\right) / \bar{l}^{2}$ as a variation of probability. To calculate $\gamma^{2}$ he applied the Monte Carlo simulation method. Kuttruff introduced two important changes to the Eyring equation. The first one involved the shape of the room, while the other one involved the distribution of the absorbing material. He also introduced a correction in determining the average sound absorption coefficient, which yielded the following equation:

$$
\begin{aligned}
T_{K U T} & =\frac{0.161 V}{S \bar{\alpha}_{K U T}}[\mathrm{~s}], \\
\bar{\alpha}_{K U T} & =-\ln \left(1-\bar{\alpha}_{S A B}\right)\left(1+\frac{\gamma^{2}}{2} \ln \left(1-\bar{\alpha}_{S A B}\right)\right),
\end{aligned}
$$

where $\gamma^{2}$ is the mean free path.

All the formulas presented above are applied to determine the reverberation time in rooms where the distribution and acoustic properties of the used materials are uniform in all directions. It means that the absorption of the opposite plane pairs (pairs of walls, ceiling and floor) limiting the room is approximately equal. In the further part of the paper we present three formulas (Fitzroy's, Arau's and Neubauer's) used to predict the reverberation time of a room, which take into account the propagation of sound in three orthogonal directions $x, y, z$. The formulas developed by Fitzroy (Fitzroy, 1959) or (ARAU-PUCHADES, 2005) take into account the uneven distribution of sound absorbing materials and systems in the room $\left(\bar{\alpha}_{x} \neq \bar{\alpha}_{y} \neq \bar{\alpha}_{z}\right)$. Thus, isotropic field condition is not fulfilled, which leads to the increase of reverberation time of the interior determined with the application of the previously discussed formulas (Sabine, Eyring, Kuttruff and Millington). Therefore, the Fitzroy's formula should be used, as it offers values that are more consistent with the values obtained in the measurement. The Fitzroy's equation has the following form:

$$
T_{F I T}=\left(\frac{S_{x}}{S}\right) \cdot T_{x}+\left(\frac{S_{y}}{S}\right) \cdot T_{y}+\left(\frac{S_{z}}{S}\right) \cdot T_{z}[\mathrm{~s}],
$$

where

$$
\begin{gathered}
T_{x}=\frac{0.161 \mathrm{~V}}{S \bar{\alpha}_{F I T, x}}, \quad T_{x}=\frac{0.161 \mathrm{~V}}{S \bar{\alpha}_{F I T, y}}, \quad T_{z}=\frac{0.161 \mathrm{~V}}{S \bar{\alpha}_{F I T, z}}, \\
\bar{\alpha}_{F I T, x}=-\ln \left(1-\bar{\alpha}_{x}\right), \quad \bar{\alpha}_{F I T, y}=-\ln \left(1-\bar{\alpha}_{y}\right), \\
\bar{\alpha}_{F I T, z}=-\ln \left(1-\bar{\alpha}_{z}\right),
\end{gathered}
$$

where $S_{x}, S_{y}, S_{z}$ - surfaces of the opposite pairs of walls $\left[\mathrm{m}^{2}\right], \bar{\alpha}_{x}, \bar{\alpha}_{y}, \bar{\alpha}_{z}$ - average reverberation sound absorption coefficients of the material on the respective pairs of walls.

Arau-Puchades (2005) proposed an improved equation which assumed that the reverberation time of the interior could be determined as a geometric weighted average of three reverberation times obtained from the orthogonal directions $(x, y, z)$. He also assumed that the decay of the reverberation time was of hyperbolic nature. The absorption coefficients used in his formula are the mean absorption values for each pair of the opposite walls. Simultaneous sound reflections are formed between these surfaces, and therefore the decay of sound should be considered in three directions. Arau-Puchades determines the reverberation time of the interior in the following way:

$$
\begin{aligned}
T_{A R A U}= & {\left[\frac{0.161 V}{S \bar{\alpha}_{A R A U, x}}\right]^{S_{x} / S} \cdot\left[\frac{0.161 V}{S \bar{\alpha}_{A R A U, y}}\right]^{S_{y} / S} } \\
& \cdot\left[\frac{0.161 V}{S \bar{\alpha}_{A R A U, z}}\right]^{S_{z} / S},
\end{aligned}
$$

where

$$
\begin{gathered}
\bar{\alpha}_{A R A U, x}=-\ln \left(1-\bar{\alpha}_{x}\right), \quad \bar{\alpha}_{A R A U, y}=-\ln \left(1-\bar{\alpha}_{y}\right), \\
\bar{\alpha}_{A R A U, z}=-\ln \left(1-\bar{\alpha}_{z}\right)
\end{gathered}
$$

ARAU-PUCHADES together with BERARd (2013) modified the non-symmetrical model $T_{A R A U}$. Knowing the expressions for the direct and diffuse sound 
pressure, it is possible to obtain a formula for the reverberation radius. This comes from equaling the sound intensity in the direct field in $I_{\text {direct }}=Q w / 4 \pi r^{2}$, where $Q$ is the source directivity, w is the sound power of the source and $r$ is the distance from the source to the receiver. And the reflected sound intensity in a diffuse field in $I_{\text {diffuse }}=4 w / A$, where $A$ is the equivalent absorption $A=[-S \ln (1-\alpha)+4 m V], \alpha$ is the absorption coefficient, and $\mathrm{m}$ is the sound absorption coefficient of the air. We obtain in effect: $r_{\mathrm{HD}}=((0.01 / \pi)(V / T))^{1 / 2}$.

From the equation $T_{A R A U}$, it is possible to calculate the reverberation radius in each direction, as in the expression $r_{\mathrm{HD}}: r_{\mathrm{HND} i}^{2} \cdot 16 \pi=A_{i}, i=x, y, z$.

In the last equation the reverberation radius in each direction increases as the equivalent absorption in that direction rises. From this, it is possible to calculate the reverberation time as a product of the terms of reverberation for the facing surfaces:

$$
\begin{aligned}
T= & \left(0.01 V / \pi r_{\mathrm{HND} x}^{2}\right)^{S_{x} / S} \cdot\left(0.01 V / \pi r_{\mathrm{HND} y}^{2}\right)^{S_{y} / S} \\
& \cdot\left(0.01 V / \pi r_{\mathrm{HND} z}^{2}\right)^{S_{z} / S}, \\
T= & \left(0.01 V / \pi r_{\mathrm{HND}}^{2}\right),
\end{aligned}
$$

where

$$
r_{\mathrm{HND}}=\left[r_{\mathrm{HND} x}^{S_{x} / S} \cdot r_{\mathrm{HND} y}^{S_{y} / S} \cdot r_{\mathrm{HND} z}^{S_{z} / S}\right]
$$

with the reverberation radii for a non-diffuse sound field $r_{\text {HND }}$ obtained as:

$$
\begin{aligned}
& r_{\mathrm{HND}}=((0.01 / \pi)(V / T))^{1 / 2}, \\
& r_{\mathrm{HND}}=(\mathbf{A} / 16 \pi)^{1 / 2},
\end{aligned}
$$

where

$$
\mathbf{A}=A_{x}^{S_{x} / S} \cdot A_{y}^{S_{y} / S} \cdot A_{z}^{S_{z} / S} .
$$

Neubauer, Kostek (2001) presented a modification of the Fitzroy's equation, dividing appropriately the Kuttruff's correction element into two parts. One part reflects the surfaces of the floor and ceiling, while the second part takes into account the impact of the other walls.

$$
T_{N E U}=\frac{0.45 V}{S^{2}}\left(\frac{l w}{\bar{\alpha}_{C F}}+\frac{h(l+w)}{\bar{\alpha}_{W W}}\right)[\mathrm{s}] .
$$

In the Neubauer's equation, the sound field is divided into two parts, where the determined absorption coefficients are treated as an adjustment to the Eyring and Kuttruff's formula:

$$
\begin{gathered}
\bar{\alpha}_{C F}=-\ln \left(1-\bar{\alpha}_{S A B}\right)+\frac{\rho_{C F}\left(\rho_{C F}-\bar{\rho}\right) S_{C F}^{2}}{(\bar{\rho} S)^{2}}, \\
\bar{\alpha}_{W W}=-\ln \left(1-\bar{\alpha}_{S A B}\right)+\frac{\rho_{W W}\left(\rho_{W W}-\bar{\rho}\right) S_{W W}^{2}}{(\bar{\rho} S)^{2}},
\end{gathered}
$$

where $l, w, h$ - length, width and height of the room $[\mathrm{m}], \bar{\alpha}_{C F}$ - average effective sound absorption coefficient of the ceiling and floor, $\bar{\alpha}_{W W}$ - average effective sound absorption coefficient of the side partitions, $\rho=1-\alpha-$ reflection coefficient, $S_{C F}-$ surface of the ceiling and floor, $S_{W W}$ surface of the side walls [m].

PuJOlle (1975) proposed another determination method of the mean free path $l_{m}$ taking into account the dimensions of the room. He presented two formulas to determine $l_{m}$ :

$$
l_{m}=\frac{1}{6}\left(\sqrt{L^{2}+l^{2}}+\sqrt{L^{2}+h^{2}}+\sqrt{h^{2}+l^{2}}\right)
$$

Or

$$
l_{m}=\frac{1}{\sqrt{\pi}}\left(L^{2} l^{2}+L^{2} h^{2}+h^{2} l^{2}\right)^{1 / 4} .
$$

The Pujolle reverberation time can be determined according to the formula:

$$
T_{P U J}=\frac{0.04 l_{m}}{\bar{\alpha}_{E Y R}}[\mathrm{~s}]
$$

where $L, h, l$ - length, height and width of the room $[\mathrm{m}]$.

According to SKRZYPCZYK (2008) and WinkLERSkAlna (2008) reverberation time can be also estimated using the perturbation numbers. They presented the application of a new algebraic system for the determination of reverberation time. They introduced the perturbations of parameters into the Sabine's equation. Assuming that all the parameters $\left(V_{P E R}, S_{P E R}, \bar{\alpha}_{S A B, P E R}\right)$ in Sabine's formula undergo $2 \varepsilon$-perturbation, we obtain:

$$
T_{S A B, P E R}=\frac{0.161 V_{P E R}}{4 m V_{P E R}+S_{P E R} \bar{\alpha}_{S A B, P E R}},
$$

where

$$
\begin{aligned}
V_{P E R} & =V_{0}+\varepsilon_{1} V_{1}+\varepsilon_{2} V_{2}, \\
S_{P E R} & =S_{0}+\varepsilon_{1} S_{1}+\varepsilon_{2} S_{2}, \\
\bar{\alpha}_{S A B, P E R} & =\bar{\alpha}_{S A B, 0}+\varepsilon_{1} \bar{\alpha}_{S A B, 1}+\varepsilon_{2} \bar{\alpha}_{S A B, 2},
\end{aligned}
$$

where $V_{P E R}, S_{P E R}, \bar{\alpha}_{S A B, P E R}$ - room volume, total surface area of the partitions limiting the room, absorption coefficient (parameters with perturbations), $\varepsilon_{i}$ - small parameter, $i=1,2$, respectively.

The inverse of the 2-perturbation number:

$$
\zeta^{-1}=(x, y, z)^{-1}=\left(\frac{1}{x},-\frac{y}{x^{2}},-\frac{z}{x^{2}}\right), \quad x \neq 0 .
$$

Thus, by using the inverse of $2 \varepsilon$-number, the reverberation time has the following form:

$$
\begin{aligned}
T_{S A B, P E R}= & \frac{0.161 V_{P E R}}{4 m V+S \bar{\alpha}_{S A B, 0}}-\frac{0.161 V S}{\left(4 m V+S \bar{\alpha}_{S A B, 0}\right)^{2}} \\
& \cdot\left(\varepsilon_{1} \bar{\alpha}_{S A B, 1}+\varepsilon_{2} \bar{\alpha}_{S A B, 2}\right) \\
= & T_{S A B, 0}+\varepsilon_{1} T_{S A B, 1}+\varepsilon_{2} T_{S A B, 2}
\end{aligned}
$$

where $T_{S A B, 0}$ - reverberation time without perturbation $[\mathrm{s}], T_{S A B, i}$ - perturbation components of the reverberation time $[\mathrm{s}]$. 
The reverberation time of a room can be also determined using the recommendations of the standard (PN EN 12354-6, 2005). The said standard reads that the room reverberation time is dependent on the volume and on the total equivalent area of the sound-absorbing interior (total acoustic absorption), as well as on the fraction of the objects:

$$
T=\frac{55.3}{c_{0}} \frac{V(1-\psi)}{A},
$$

where $c_{0}$ - speed of sound in the air, $A$ - equivalent area of the sound-absorbing interior, referred to as the acoustic absorption of the interior, $\psi$ - fraction of objects defining the volume of the objects in the room.

The total acoustic absorption can be calculated as follows:

$$
A=\sum_{i=1}^{n} \alpha_{s, i} S_{i}+\sum_{j=1}^{o} A_{o b, j}+\sum_{k=1}^{p} \alpha_{s, k} S_{k}+A_{i r},
$$

where $n$ - number of surfaces $i, o$ - number of objects $j, p$ - number of $k$-system objects.

The equivalent surface area for air absorption is determined basing on the formula $A_{\text {air }}=4 m V(1-\psi)$. This standard reads that the air absorption may be omitted if the room has the volume of less than $200 \mathrm{~m}^{3}$ and the calculations are carried out to $1000 \mathrm{~Hz}$ octave band as the highest frequency. The fraction of objects was determined from the following equation:

$$
\psi=\frac{\sum_{j=1}^{o} V_{o b j, j}+\sum_{k=1}^{p} V_{o b j, k}}{V},
$$

where $V_{o b j}$ - volume of hard objects.

Due to some limitations, this method can be applied for rooms of regular-shape (none of the interior dimensions can be five times greater than the others), with a small number of objects which are also characterized by uniform sound distribution. If the above conditions are not met, the obtained values of room reverberation time can differ from the estimated ones.

\section{Investigation studies on reverberation time}

Using the commonly available technical data involving room acoustics, the verification of the usefulness of the theoretical models of reverberation time was carried out in terms of their applicability to different rooms. In the available articles, the authors compare their new methods for the prediction of reverberation time with the most commonly applied formulas of Sabine, Eyring, Millington-Sette and Fitzroy. The results of the analytical calculations are often compared with the values obtained from computer simulations and actual measurements. The world literature offers many publications helpful to verify the usefulness of theoretical models used for the estimation of reverberation time.
In the work (Neubaer, Kostek, 2001), the authors presented a new formula for the calculation of reverberation time and compared it to other existing formulas by Sabine, Eyring, Millington-Sette, Fitzroy, Tohyama (1986), Arau as well as to a new formula (Neubauer) and model incorporated in the European standard (Annex D of the proposition of EN 123546,2003 ) along with the measurements. First, the researchers analyzed two extreme cases of rooms having regular shapes. In the first room (alive) they used materials of low absorption $(\alpha=0.02)$, while in the second one (dead), the average absorption coefficient of the surface was $(\alpha=0.95)$. Then, the authors examined the room with the unevenly distributed acoustic absorption. In that case, the floor and ceiling were characterized by higher absorption coefficient $(\alpha=0.43)$, while the other areas had low absorption $(\alpha=0.02)$. In effect of the carried out research studies, the authors arrived at the following conclusions:

1) the reverberation time obtained from the standard formula, by Tohyama and Fitzroy, is significantly different from the measured values;

2) when the room has a low absorption coefficient, there are no significant differences between the measured values, except for the formula included in the standard (Annex D of the proposition of EN 12354-6, 2003) and Tohyama's formula;

3) the new formula is more effective in determining the reverberation time than the classical formulas.

In the next work (KANG, NEUBAER, 2001), the authors compare the analytical formulas and computer simulations performed by means of the CATTAcoustic software (CM) and radiosity model (RM). They analyzed two rectangular rooms in which the length and width equaled $10[\mathrm{~m}]$, while the height was variable -8 or $3[\mathrm{~m}]$. They examined 8 cases involving the distribution of absorbing material on the surfaces limiting the room. For the analysis they used two different absorbing surfaces, with the absorption coefficient $(\alpha=0.08)$ for the surfaces marked with gray color, and with the absorption coefficient $(\alpha=0.05)$ for the remaining ones. In the present paper the authors arrived at the following conclusions:

1) for the room with a constant absorption coefficient ( $\alpha=0.05)$ on all limiting surfaces, they received similar values using the classical formulas (case 1);

2) for the remaining cases they received high reverberation times for the Fitzroy's, Arau's and standard formulas;

3) the Fitzroy's formula had the highest result, and the Millington's formula the lowest;

4) the values obtained from the calculations performed with the use of the standard formula and Arau's formulas are similar;

5) they also observed that the values obtained from CM were higher than those from RM; 
6) the radiosity model generates the average reverberation time close to the values obtained from the Fitzroy's and Kuttruff's formulas.

Another approach to the estimation of reverberation time was presented by ZHANG (2005). In his dissertation he suggested a new model for the prediction of reverberation time and also defined the deviation between the measurement and the expected $T$ value. On the basis of the obtained results Zhang provided the following conclusions:

1. The Fitzroy's method reached the greatest deviation of 12.9 .

2. For high frequencies, the Kuttruff's equation is applicable whereas for low frequencies the ArauPuchade's formula is applicable.

3. It was also demonstrated that the model (Zhang) had the smallest deviation equal to 0.22 .

The estimation of reverberation time has been developed not only on the basis of empirical formulas, but also on the basis of the commonly known mathematical methods, based on finite element methods, perturbations or neural networks. Using one of these methods, SKRZYPCZYK (2008) and WINKLER-SKALNA (2008) proposed a new determination method of reverberation time. The authors presented the methods which make use of the new perturbation algebra for the analysis of acoustic problems in enclosed rooms. They introduced an assumption advocating that all parameters in the Sabine, Eyring and Kuttruff formulas undergo $2 \varepsilon$-perturbation. Basing on the above, they presented modified classical formulas for the determination of reverberation time. In his work, SKRZYPCZYK (2010) presented the results of the reverberation time calculations in Aula Magna and in the Olivertani Church determined according to the Eyring's formula. Basing on the achieved results, the author provided the following conclusions:

1. The results of the calculations made with the use of the Eyring's and Sabine's perturbation formulas coincide for small-sized perturbations.

2. When only one parameter is subjected to perturbation, the perturbation calculations are compatible with the actual perturbation in the whole range.

Despite the fact that the classical formulas have been attributed to simple objects and objects with uniform absorption, some authors attempted to find a correlation between the results of reverberation time according to the already mentioned formulas for complex objects. In the paper (IORDACHE et al., 2013), which was to determine the applicability of Sabine's formula in the rooms of complex shape, the authors studied a room consisting of two levels. The height of each level was $4.5 \mathrm{~m}$ and the volume of the whole room was $2926 \mathrm{~m}^{3}$. Basing on the obtained results, they observed the differences between the value obtained from the classical formula and from the measurement. The value determined from the Sabine's formula was by $0.5 \mathrm{~s}$ higher than that obtained from the measurement. The authors of the publication explained that the difference could have been effected by the following reasons. Firstly, errors in the measurement of the surfaces limiting the room due to complex shape of the room. Secondly, erroneously employed material absorption coefficients. Thirdly, they questioned the use of the Sabine's formula for that type of room. Summing up the results obtained in the course of the studies, they concluded that the Sabine's formula should not be applied to the interiors of complex shape during the design phase.

As in the publication (IORDACHE et al., 2013), the authors (KANG et al., 2007) analyze the calculation methods of reverberation time in large spaces (atrium). The research found that the reverberation times obtained using the Sabine's formula were comparable to the average value obtained from the measurement for four receivers. However, when they compared the individual results at each point, they were different by $15 \%$ to $23 \%$. They assumed in the analysis conducted in such a way that the classical formula might be used in the estimation process of average reverberation time for the entire interior.

The research in the field of acoustics did not focus solely on the errors in the formulas for the estimation of reverberation time. The authors analyzed also the impact of two standard absorption coefficients and one determined from the Millington's formula with respect to the mentioned parameter. In the work (PETELJ et al., 2012), the authors compare the reverberation times obtained from the classical formulas where standard sound absorption coefficients were used with the formula given by Millington. In the Millington's formula they used his sound absorption coefficients. The measurements were carried out in two rooms of irregular shape. The first tested room had the floor surface of $32.6 \mathrm{~m}^{2}$ and height of $3.25 \mathrm{~m}$, while the second one the floor surface of $14.15 \mathrm{~m}^{2}$ and height of $2.8 \mathrm{~m}$. The research studies in the first empty room yielded the average value of reverberation time equal to $T=4 \mathrm{~s}$. In effect of the theoretical works, they obtained the value $T=3.99 \mathrm{~s}$ from the Millington's formula and $T=4.11 \mathrm{~s}$ determined on the basis of the Zhang method. The calculations carried out on the basis of the Sabine's formula yielded the value $T=4.88 \mathrm{~s}$. The best estimate was obtained for the first example of Millington's formula. In the next room they received the average value of reverberation time of the in situ research equal to $T=1.21 \mathrm{~s}$. From the classical formulas they obtained the value of reverberation time for Sabine $T=1.26 \mathrm{~s}$, Millington $T=1.15 \mathrm{~s}$ and Zhang $T=1.20 \mathrm{~s}$, respectively. 
The authors presented the following conclusion: the Millington's formula yielded results with small deviations, but only when the average reverberation time was determined for the frequency range from $125 \mathrm{~Hz}$ to $4 \mathrm{kHz}$.

Also the works (DANCE, SHIELD, 1999; 2000) compare the formulas which apply the Millington and standard absorption coefficients. In the first publication, the authors analyzed the application of the standard and Millington's absorption coefficients and compared the applied classical formulas. Research studies were also carried out in a recording studio and concert hall in order to estimate the accuracy of the reverberation time with the use of Millington's formula and his absorption coefficients. Then, the obtained values were compared with those obtained on the basis of Sabine's and Eyring's formulas. Having analyzed the results of the measurements and the prediction of reverberation time based on the Sabine's, Eyring's and Millington's formulas in the recording studio, they found that the formula suggested by Millington was as accurate as the classical formulas. Afterwards, the authors determined the errors yielded by the formulas. The error for the Millington's formula was $10.9 \%$ for all frequencies, while for the Sabine's formula $12.8 \%$ and Eyring's 20\%. Then, they conducted a study in a concert hall. Summing up the results of their works, they observed significant differences between the obtained results. They found that the classical formulas were becoming less accurate for rooms with unevenly distributed field. In the second paper, the Sabine's and Millington's formulas were used to analyze which one was more useful. The research involved an experimental room $(4.54 \times 2.73 \times 2.4 \mathrm{~m})$ with differently situated sound-absorbing material.

The authors compared the results of the calculations made with the use of different formulas of reverberation time to the values obtained by means of the measurement. The authors drew the following conclusions:

1. For the case 0 they obtained (by means of Sabine's, Eyring's, Millington's and Arau's formulas) the reverberation time values with the error within the range of $6 \%$.

2. The Arau's formula for the cases $1-5$ had the error of $8.3 \%$ in comparison to other methods which had the errors of $42 \%, 31 \%$ and $33 \%$ for the Eyring's, Sabine's and Millington's formulas respectively.

3. The above results illustrate the applicability limits of the classical methods, except for the Arau's formula.

Lawrence presented in his dissertation (LAWRENCE, 2006) a problem involving the estimation of sound absorption coefficient basing on the Sabine's and Eyring's formulas. The author outlined the problem involving the determination of the coefficient $\alpha$ based on the Sabine's formula which implied that the absorption exceeding $100 \%$ was possible. Referring to other scientists, he presented the problems related to the measurement of the absorption coefficient. The publication also cites HoDGSON's (1993) statement which reads that the Eyring's formula is more accurate to estimate the reverberation time and to determine the absorption coefficient. Despite many discussions and various studies presented in papers, none of these publications leads to a solution, but only underlines the significance of the problem.

The problem involving the determination of the absorption coefficient was also referred to by BERANEK (2006). Having analyzed the examples, he found that the Sabine's formula could be used to determine the reverberation time in the room for which the coefficient $\alpha$ had been earlier determined in a similar location.

A considerable part of the publication presented the analysis involving the impact of the extent of room filling as well as the impact of the unevenly distributed absorbing material. In another study (PAssero, ZANNIN, 2010), the authors attempted to show and verify the similarity of procedures for the determination of reverberation time in a classroom. In the publication PAssero, ZANNIN (2010) presented the statistical analysis of data obtained from the comparison of all applied methods. Basing on the data included in that study, the authors presented the following conclusions:

1. Lack of differences between the measurement methods, which was also confirmed by the Astolfi's publication (AsTOLFI et al., 2008).

2. The results of the study show that the Eyring's formula is the most similar to the two measurements and to the computer simulation of all the models used, while the Arau's formula is the least similar.

3. The reverberation time obtained from the Sabine's formula was longer than that calculated according to Eyring, which was also reported in the work (BISTAFA, BRADLEY, 2000).

The work (Wilmshurst, ThOMPson, 2012) shows the reverberation time prediction methods for rectangular rooms with unevenly distributed absorbing material. The authors suggested a different way of determining the reverberation time using the Statistical Energy Analysis method (SEA) and compared the classical methods with the computer simulations carried out with the use of CATT-Acoustic software. The SEA model calculates the total potential energy of sound in connection with the time function, and on that basis a theoretical graph of sound decay is obtained. In the analyzed room of the dimensions $10 \times 9 \times 8 \mathrm{~m}$, they placed absorbing materials on different surfaces limiting the interiors and checked the usefulness of the used calculation methods. Basing on the values obtained from all methods, the authors presented the following conclusions: 
1. The SEA model yielded values close to those of CATT-Acoustic and Arau.

2. In the case of a non-homogeneous room (ceiling, floor and side walls are lined with absorbing material), the Fitzroy's formula and SEA model yielded the highest value of reverberation time.

Also I. Rossell and I. Arnet studied a similar problem, taking into account the audience in the Hall (Rossell, Arnet, 2002). The authors presented in their publication the results of reverberation time for three cases of hall filling (in an empty classroom, with $50 \%$ filling and filled in 100\%). Basing on the results obtained for all determination methods of reverberation time, the authors summarized the work with the following conclusions:

1. For the first situation (no audience) the obtained reverberation time values are comparable, with the exception of Fitzroy's equation. In that case we have a diffused field in which the classical theories yield good results.

2. For the room with $50 \%$ filling, the results obtained from the theoretical calculations differ from the measurement values.

3. The values obtained from the Arau's formula proved to be the most accurate in estimating the reverberation time. Summarizing the analyses of the material collected from the literature, it can be concluded that the current formulas used to calculate the reverberation time in a room have large deviations from the actual values, and in particular for cuboid objects with non-diffusive (dispersed) sound field. The objects of that type are most frequently met in practice. For that type of objects, the acoustic properties of a room or absorption values applied in the design process should be more accurate than the values having a high degree of approximation.

In recent times, many publications involve room acoustics by means of computer simulations. In the authors' opinion two works can be quoted as the most interesting. BERARDI (2014) presented in his paper the results of computer simulations involving the acoustics of box-shaped churches. During the simulation he was changing the ratios of width, length and height of the buildings. Basically, he was analyzing the dependence between the selected acoustic parameters and the shape of the object. He presented equations describing the acoustics of the simulated churches and then he verified the obtained results with the measurements carried out in five Italian churches.

In the work of Bustamante, Girón, Zamarreño (2014) simulation techniques have been implemented to study the sound fields of a multi-configurable performance enclosure by creating computer acoustic 3Dmodels for each room configuration.
The digital models have been tuned by means of an iterative fitting procedure that uses the reverberation times measured on site for unoccupied conditions with the orchestra shell on the stage.

The most interesting works quoted here by the authors contain references to other sources which are also worth attention.

\section{Case study}

For the needs of the case study in an anechoic room, a cuboid room of the floor dimensions $2.5 \times 5.0 \mathrm{~m}$ and height $2.5 \mathrm{~m}$ made of OSB was built. Then, reverberation time was measured for such a structure in two variants. The first variant involved an empty room built from the boards having the sound absorption index lower than 0.2. The second variant involved the same room but with disturbed acoustic field in the form of mineral wool placed on the partitions limiting the room (see Fig. 1).

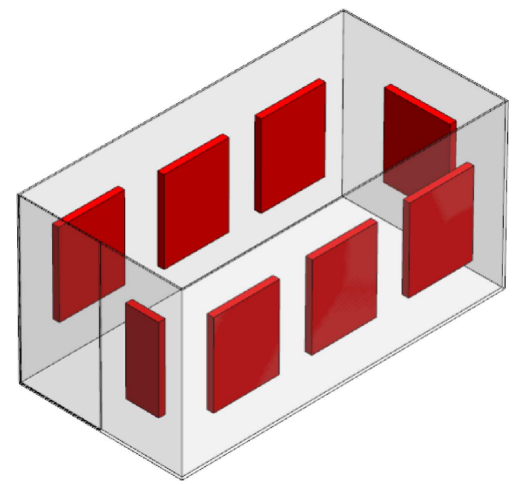

Fig. 1. Location of sound absorbing material in the model room.

The investigation of reverberation time consisted in measurements and theoretical calculations with the application of equations described in this paper. The results are presented on the diagrams below.

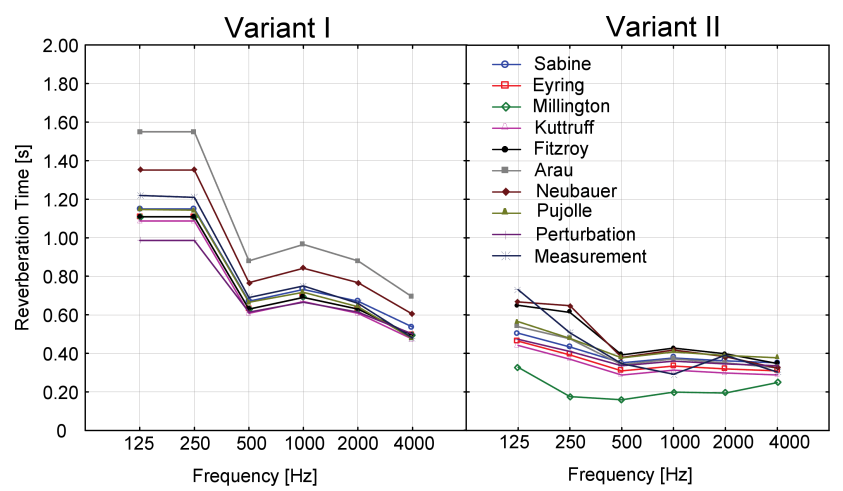

Fig. 2. Measured and calculated reverberation times in the analyzed variants.

Basing on such a simple analysis, we can see a difference between the measurement and theoretical 
equations. The results are different for the room with uniformly distributed sound absorption index and different for the rooms with sound absorption elements. This simple case study confirms the observations of many researchers which have been presented in the previous sections of this paper.

Case studies have been also carried out by many other researchers as for example McMinn (1996). In his work he investigated among other things the Musikvereinssaal Hall in Vienna which is one of the biggest concert halls in the world (see Fig. 3).

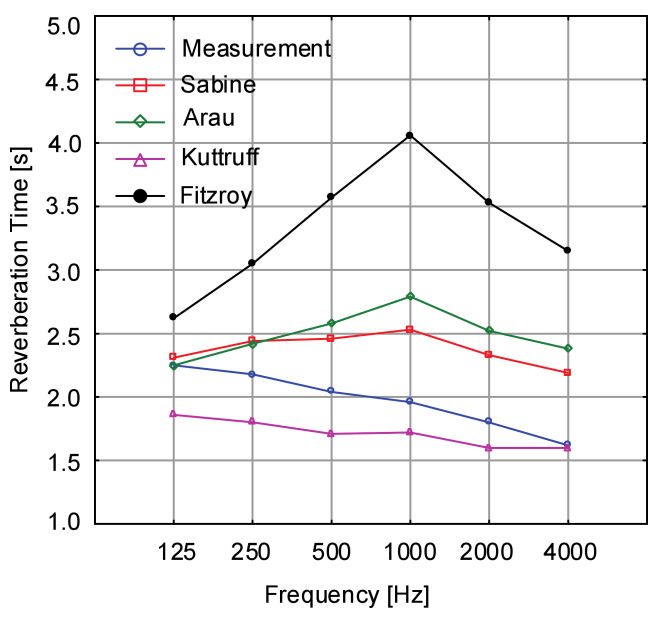

Fig. 3. Grosser Musikvereinssaal Hall (MCMinn, 1996).

The research results provided by McMinn also confirm the observations of many researchers quoted in this paper. It is also worthwhile mentioning another work (NEubAUER, KosteK, 2001) in which other cases have been analyzed, e.g. the work presenting reverberation time as a function of the volume of the poorly damped room, with reverberation times being calculated with the application of different equations.

Such an approach was employed to show the variation of the obtained results.

Interesting research studies were published by KraszewsKi (2012). In his paper he presented theoretical calculations for long rooms. He observed in his conclusions that the reverberation time was influenced not only by the mean absorption index but also by the location of the absorbing material or the place at which the reverberation time was calculated by means of the diffusion equation.

\section{Application of reverberation time for the description of room acoustics}

As it has been already mentioned in the Introduction of the present paper, reverberation time can influence other parameters used to design rooms of various types. In this section we will only signal the results of some selected works.
LAM (1999) provided the approximations of certain parameters by means of reverberation time, such as:

Definition (Deutlichkeit): $D_{50}=1-e^{-\frac{0.69}{T_{60}}}$;

Clarity: $C_{80}=10 \log \left(e^{\frac{1.1}{T_{60}}}-1\right)$;

Centre Time: $T_{c}=\frac{T_{60}}{13.8}$;

Level: $L=10 \log \left(\frac{16 \pi 10^{2} T_{60}}{0.161 T}\right)$.

The authors of this article NowoświAT and OLECHOWSKA (2016) provided the approximation of speech transmission index by means of reverberation time: $\mathrm{STI}=A \ln +B$, where $A=-0.2078, B=0.6488$.

ARETz and OrLOWSKI (2009) indicated in their measurements that sound strength can be lowered by means of an appropriate shaping of reverberation time. On the other hand, to ensure proper reception of chamber music, reverberation time cannot be lowered freely. Therefore, they suggested that the reverberation time and sound strength should be balanced. Basing on the least squares method, they worked out a regression model

$$
\begin{aligned}
G_{\text {reflected }} & =10 \cdot \log _{10}((31200 R T / V) \cdot b) \\
& =10 \cdot \log _{10}(31200 R T / V)+\widehat{b}
\end{aligned}
$$

Then, the authors state that between $G_{\text {reflected }}$ and $10 \cdot \log _{10}(31200 R T / V)$ we can observe high correlation $r=0.94$ for $\widehat{b}=-3.3 \mathrm{~dB}$ with the standard deviation of $1.1 \mathrm{~dB}$.

\section{Conclusion}

The review of the literature shows that the existing and widely used formulas for the determination of reverberation time do not allow us to predict accurately the reverberation time in the interiors filled with audience (currently, too large differences have been observed as compared to the actual values, measured while determining the reverberation time). Designers involved in shaping the acoustic properties of rooms are looking forward to the development of a formula which would more precisely determine the prediction of reverberation time. This paper reviews the available literature in the field concerned with the assessment and verification of the usefulness of the applied models designed to determine reverberation time of a room.

The volume of the present publication indicates that a great number of researchers are still working on the estimation of reverberation time. A lot of them create new formulas whereas others verify them. With respect to many works, it is not possible to decide explicitly which formula is the best. To provide an example, we can quote the research carried out by PASSERO and ZANNIN (2010) in which the reverberation time in a classroom was analyzed. They used for that purpose the calculations with the formulas of Sabine, Eyring 
and Arau-Puchades. Their work demonstrated, as in the case of Bistafa (BIstafA, BRADley, 2000), that the reverberation time calculated with the Sabine formula was slightly longer than that calculated with the Eyring formula. And it was considerably longer when the Arau-Puchades formula was applied. The obtained reverberation time by means of Eyring formula was similar to the measurement and computer simulation in ODEON. In variant I the calculations by means of Sabine and Eyring formula yield the values relatively close to the measurement. The result provided by Kuttruff formula yields a considerably shorter reverberation time, and using the Arau-Puchades formula, it is considerably longer. It differs slightly in variant II where the tested room is damped. In that case, the formula of Arau-Puchades yields results which are not considerably different from other results. Much higher variation in the obtained results can be observed in the analysis of Grosser Musikvereinssaal Hall, where the Albine formula yields shorter reverberation time than that of Arau-Puchades but longer than the measurement. A novel approach was presented by Lam who described the basic parameters of room acoustics, such as Deutlichkeit, Clarity, Centre Time, Level by means of reverberation time.

In the same way the authors (Nowoświat, OLECHOWSKA, 2016) estimated the speech transmission index by means of reverberation time.

The presented research studies carried out by different research teams have prompted us to develop our own model based on the statistical theory and trend function. The model developed by the authors of this work will be described in the next publication and it will be based on "learning" algorithms and trend functions (Nowoświat et al., 2016).

\section{References}

1. Arau-Puchades H. (1988), An Improved Reverberation Formula, Acustica, 65, 163-180.

2. Arau-Puchades H. (2005), Are the scattering and the absorption coefficients two faces of a same coin? Reverberation time in two cases analyzed, International Congress on Noise Control Engineering (Internoise 2005), Rio de Janeiro, Brazil 7-10 August 2005, Vol. 1-5, ISBN 978-1-62276-339-9, 3203-3211.

3. Arau-Puchades H., Berardi U. (2013), The reverberation radius in an enclosure with asymmetrical absorption distribution, Procedings of Meetings on Acoustics, 19, 015141.

4. Aretz M., Orlowski R. (2009), Sound strength and reverberation time in small concert halls, Applied Acoustics, 70, 1099-1110.

5. Astolfi A., Corrado V., Griginis A. (2008), Comparison between measured and calculated parameters for the acoustical characterization of small classrooms, Applied Acoustics, 69, 966-976.

6. Beranek L.L. (2006), Analysis of Sabine and Eyring equations and their application to concert hall audience and chair absorption, J. Acoust. Soc. Am., 120, 3, 1399 $-1410$.

7. Berardi U. (2012), A Double Syntethic Index to Evaluate the Acoustics of Churches, Archives of Acoustics, 37, 4, 521-528.

8. Berardi U. (2014), Simulation of acoustical parameters in rectangular churches, J. of Building Performance Simulation, 7, 1, 1-16.

9. Bistafa S.R., Bradley J.S. (2000), Reverberation time and maximum background noise level for classrooms from a comparative study of speech intelligibility metrics, J. Acoust. Soc. Am., 107, 2, 861-875.

10. Bistafa S.R., Bradley J.S. (2000), Predicting reverberation times in a simulated classroom, J. Acoust. Soc. Am., 108, 1721-1731.

11. Bustamante P., Girón S., Zamarreño T. (2014), Simulated Sound-Fields in a Multi-Configurable Auditorium, Archives of Acoustics, 39, 3, 365-383.

12. Dance S.M., Shield B.M. (1999), Modeling of sound fields in enclosed spaces with absorbent room surfaces. Part I: performance spaces, Applied Acoustics, 58, 118.

13. Dance S.M., Shield B.M. (2000), Modeling of sound fields in enclosed spaces with absorbent room surfaces. Part II. Absorptive panels, Applied Acoustics, 61, 373384.

14. Engel Z., Kosata K. (2007), Index method of the acoustic quality assessment of sacral buildings, Archives of Acoustics, 32, 3, 3-22.

15. En 12354-6: 2003 Estimation of acoustic performance of buildings from the performance of elements - Part 6: Sound absorption in enclosed spaces.

16. Eyring C.F. (1930), Reverberation time in "dead" rooms, J. Acoust. Soc. Am., 1, 217-241.

17. Fitzroy D. (1959), Reverberation formula which seems to be more accurate with nonuniform distribution of absorption, J. Acoust. Soc. Am., 31, 893-897.

18. Galbrun L., Kitapci K. (2014), Accuracy of speech transmission index predictions based on the reverberation time and signal-to-noise ratio, Appl. Acoust., 81, $1-14$.

19. HiRATA Y. (1979), Geometrical acoustics for rectangular rooms, Acustica, 43, 4, 247-252.

20. Hodgson M. (1993), Experimental evaluation of the accuracy of the Sabine and Eyring theories in the case of non-low surface-absorption, J. Acoust. Soc. Am., 94, $2,835-840$. 
21. Houtgast T., Steeneken H.J.M. (1985), A review of the MTF concept in room acoustics and its use for estimating speech intelligibility in auditoria, J. Acoust. Soc. Am., 77, 3, 1069-1077.

22. Houtgast T., Steeneken H.J.M. (1984), A MultiLanguage Evaluation of the RASTI - Method for Estimating Speech Intelligibility in Auditoria, Acustica, 54, 4, 185-199.

23. Iordache V., Catalina T., Cucu B-M. (2013), Experimental Investigation of the Reverberation Time Inside a Complex Geometry Indoor Space, Romanian Journal of Acoustics and Vibration, X, 2, 109-114.

24. ISO 17497-1 (2004), Acoustics - Measurement of the sound scattering properties of surfaces, Part 1: Measurement of the random-incidence scattering coefficient in a reverberation room.

25. Kang J., Neubauer R.O. (2001), Predicting reverberation time: Comparison between analytic formulae and computer simulation, Proceedings of the 17th International Conference on Acoustics (ICA).

26. Kang J., Yap P.L., Meng Y., Chen B. (2007), Acoustics in large atrium spaces, 14th International Congress on Sound and Vibration.

27. Knudsen V.O. (1929), The hearing of speech in auditoriums, J. Acoust. Soc. Am., 1, 56-82.

28. Kraszewski J. (2012), Computing Reverberation Time in a 3D Room Model Using a Finite Difference Method Applied for the Diffusion Equation, Archives of Acoustics, 37, 2, 171-180.

29. Kuttruff H. (2009), Room Acoustics, Fifth Edition, London: Spon Press, ISBN 10: 0-203-87637-7, pp. 374.

30. LAm Y.W. (1996), The dependence of diffusion parameters in a room acoustics prediction model on auditorium sizes and ahapes, J. Acoust. Soc. Am., 100, 4, 2193-2203.

31. LAM Y.W. (1999), Importance of early energy in Room Acoustics, AEOF3/AEOF4, Acoustics of Enclosed Spaces, University of Salford, 10-28.

32. Lawrence T. (2006), The Effect of Partially Diffuse Sound Fields on the Prediction of Absorption Coefficients, MSc Audio Acoustics Dissertation, pp. 66.

33. McMinn T. (1996), Development of an Evaluation Tool for Use at the Design Stage of Auditoria with Respect to Unassisted Speech Reinforcement, Msx Thesis of the Curtis University of Technology.

34. Millington G. (1932), A modified formula for reverberation, J. Acoust. Soc. Am., 4, 69-82.

35. Neubauer R.O., Kostek B. (2001), Prediction of the Reverberation Time in Rectangular Rooms with NonUniformly Distributed Sound Absorption, Archives of Acoustics, 26, 3, 183-201.
36. Norris R.F., Andree C.A. (1930), An Instrumental Method of Reverberation Measurement, J. Acoust. Soc. Am., 1, 3, 366-372.

37. Nowoświat A., Olechowska M. (2016), Fast estimation of speech transmission index using the reverberation time, Appl. Acoust., 102, 55-61.

38. Nowoświat A., Olechowska M., Ślusarek J. (2016), Prediction of reverberation time using the residual minimization method, Appl. Acoust., 106, 42-50.

39. Ozimek E., Rutkowski L. (1985), Deformation of amplitude modulated signals (AM) propagating in a room, Architectural Acustics, Strbske Pleso, 2, 174177.

40. Passero C.R.M., Zannin P.H.T. (2010), Statistical comparison of reverberation times measured by the integrated impulse response and interrupted noise methods, computationally simulated with ODEON software, and calculated by Sabine, Eyring and Arau-Puchades' formulas, Applied Acoustics, 71, 1204-1210.

41. Petelu A., Hadžistević M., Antić A., Hodolič J. (2012), Determination of absorption coefficient of sample under non-laboratory conditions, Journal of Production Engineering, 15, 2, 75-78.

42. Plomb R., Steeneken H.J.M., Houtgast T. (1980), Predicting speech intelligibility in rooms from the Modulation Transfer Function II. Mirror image computer model applied rectangular rooms, Acustica, 46, $60-72$.

43. PN-EN 12354-6 (2005), Building Acoustic-Estimation of Acoustic Performance of Elements - Part 6: Sound Absorption In Enclosed Spaces.

44. Pujolle J. (1975), New formula for the length of time of reverberation [in French: Nouvelle formule pour la durée de réverbération], Rev. d'Acoust, 19, 107-113.

45. Rossell I., Arnet I. (2002), Theoretical and practical review of reverberation formulae for rooms with non homogenyc absorption distribution, Sevilla, Spain, Proc. Forum Acusticum.

46. SABine W.C. (1922), Collected papers on acoustics, Cambridge (MA), Harvard University Press, pp. 279.

47. Sakuma T. (2012), Approximate theory of reverberation in rectangular rooms with specular and diffuse reflections, J. Acoust. Soc. Am., 132, 4, 2325-2336.

48. Sette W.J. (1933), A new reverberation time formula, J. Acoust. Soc. Am., 4, 193-210.

49. SkrzyPCZYK J. (2010), Perturbation methods. First new algebraic methodology. Use in the mechanics an acoustics, Gliwice: Silesian University of Technology, ISBN 978-83-7335-720-4, pp. 208.

50. SKRZYPCZYK J. (2008), Perturbation methods for acoustic systems with interval parameters, Archives of Acoustics, 33, 4 (Suplement), 165-170. 
51. Tohyama M., Suzuki A. (1986), Reverberation Time in an Almost-Two-Dimensional Diffuse Field, J. Sound Vib., 111, 3, 391-398.

52. Wang L.M., Rathsam J. (2008), The influence of $a b$ sorption factors on the sensitivity of a virtual room's sound field to scattering coefficients, Appl. Acoust., 69, 1249-1257.

53. Wilmshurst L., Thompson D. (2012), A method for predicting the reverberation time and decay curves of rectangular rooms with non-uniform absorption distri- bution using Statistical Energy Analysis, Proceedings of the Acoustics Nantes Conference, 1435-1440.

54. Winkler-Skalna A. (2008), Propagation of sound waves in uncertain environment - New interval perturbation methodology, Archives of Acoustucs, 33, 4 (Suplement), 171-176.

55. Zhang Y.A. (2005), A Method to Predict Reverberation Time in Concert Hall Preliminary Design Stage, A Dissertation, Georgia Institute of Technology, December 2005, pp. 163. 